\title{
Antitumor effects of hsa-miR661-3p on non-small cell lung cancer in vivo and in vitro
}

\author{
JINGJING LU ${ }^{1 *}$, XIA GU $^{1 *}$, FANGLEI LIU ${ }^{1 *}$, ZHUANGHUA RUI $^{1}$, MING LIU $^{2}$ and LIMING ZHAO ${ }^{1}$ \\ ${ }^{1}$ Department of Respiratory Medicine, Shanghai East Hospital, Tongji University School of Medicine, \\ Pudong, Shanghai 200120; ${ }^{2}$ Department of Thoracic Surgery, Shanghai Pulmonary Hospital, \\ Tongji University School of Medicine, Yangpu, Shanghai 200082, P.R. China
}

Received July 23, 2018; Accepted March 4, 2019

DOI: $10.3892 /$ or.2019.7084

\begin{abstract}
Lung cancer is the most common and lethal cancer worldwide, especially in developing countries. Non-small cell lung cancer (NSCLC) accounts for $85 \%$ of all cases of lung cancer. In aprevious study, the protein expression of ubiquitin conjugating enzyme E2 C (UBE2C/UbcH10) in NSCLC tissues and cells was found to be significantly higher than that in adjacent tissues and normal lung epithelial cells. Further study revealed that the aberrant expression of $\mathrm{UbcH} 10$ in NSCLC tumors or cancer cells was caused by inactivation of the post-transcriptional regulation mechanism, and thus microRNAs (miRNAs) may play an important. In the present study, it was demonstrated that the expression of microRNA, hsa-miR661-3p, was downregulated and UbcH10 was upregulated in 12 pairs of NSCLC tumors and three NSCLC cell lines. A reporter gene assay revealed that overexpression of hsa-miR661-3p effectively reduced the activity of luciferase expressed by a vector bearing the $3^{\prime}$ untranslated region of UbcH10 mRNA. Ectopic hsa-miR661-3p overexpression mediated by lentiviral infection decreased the expression of UbcH10. Infection of Lv-miR661-3p inhibited cell growth and invasion in A549 and SK-MES-1 cells. Mechanistically, hsa-miR661-3p induced cell cycle G2 arrest through regulation of spindle assembly checkpoint (SAC) function. On the basis of the proposed mechanisms, the objective of the study was to inhibit the proliferation of A549 and SK-MES-1 by expressing
\end{abstract}

Correspondence to: Dr Liming Zhao, Department of Respiratory Medicine, Shanghai East Hospital, Tongji University School of Medicine, 1800 Yuntai Road, Pudong, Shanghai 200120, P.R. China E-mail: zhao.liming@hotmail.com

Dr Ming Liu, Department of Thoracic Surgery, Shanghai Pulmonary Hospital, Tongji University School of Medicine, 507 Zhengmin Road, Yangpu, Shanghai 200082, P.R. China

E-mail: liumingvip@hotmail.com

*Contributed equally

Key words: hsa-miR661-3p, UbcH10, NSCLC, SAC, proliferation, invasion
hsa-miR661-3p in vivo and in vitro. Collectively, our results indicated that downregulation of hsa-miR661-3p was involved in NSCLC and restoration of hsa-miR661-3p impaired the growth of NSCLC cell lines A549 and SK-MES-1, suggesting that hsa-miR661-3p may be a potential target molecule for the therapy of NSCLC.

\section{Introduction}

Dysfunction of cell cycle progression plays a key role in tumor formation and progression, and abnormally regulated cell cycle progression often leads to tumorigenesis and progression $(1,2)$. As one of the many genes regulating cell cycle progression, $\mathrm{UbcH} 10$ overexpression has been found in tumor tissues from a variety of sources and its overexpression has been revealed to be correlated with tumor histological grade $(3,4)$, cell proliferation (5) and poor patient prognosis $(6,7)$. In recent years, we focused on the function of UbcH10 in NSCLC. Based on the available data, the expression of UbcH10 in NSCLC was similar to the level in other types of tumors. A significant positive correlation with tumor pathological grade and tumor cell proliferation was noted. The data revealed that UbcH10 may play a key role in the pathogenesis of NSCLC. Our previous study revealed that $\mathrm{UbcH} 10$ controls excessive proliferation of NSCLC cells through the regulation of tumor protein p53 (P53) and Ki-67 and other proliferation-related genes, while UbcH10 knockdown can significantly inhibit the proliferation of NSCLC cells, which causes cell cycle arrest and promotes apoptosis. On the other hand, high expression of UbcH10 was found to reduce the sensitivity of NSCLC cells to chemotherapeutic drugs by promoting the expression of multi-drug resistance (MDR1) gene, while the targeted knockdown of the UbcH10 gene could effectively enhance chemotherapy sensitivity of SK-MES-1 and A549 cell lines to gemcitabine and paclitaxel (8).

The fundamental purpose of gene function research is to systematically elucidate the upstream regulatory mechanisms. By following the study on the regulatory mechanism, we can effectively prevent NSCLC from a macro perspective, and develop effective personalized treatment plans for patients (9-11). In essence, the root of cancer development involves epigenetics. Epigenetics can control gene expression through regulation of DNA methylation, histone modification, 
chromatin remodeling and non-coded RNA, and run through the whole process of cancer occurrence and development (12). Therefore, the in-depth study of cancer epigenetics has important significance for the clinical diagnosis, treatment and prevention of cancer. In the present study, we focused on the mechanism of the abnormal expression of $\mathrm{UbcH} 10$ in NSCLC. We found that the direct cause of the abnormal post-transcriptional regulatory mechanism of $\mathrm{UbcH} 10$ was the decrease in hsa-miR661-3p expression. Investigation of epigenetic research data was not the primary goal of present study. In the future, we are planning to explain the cause of the aberrant expression of hsa-miR661-3p in NSCLC from the focus of epigenetics. Therefore, we demonstrated the mode of action and functional specificity of hsa-miR661-3p/UbcH10 in the regulatory pathway of lung cancer through a complete and logical experimental design. On this basis, we carried out a preliminary validation of in vitro and in vivo inhibition experiments for non-small cell lung cancer by the aberrant expression of hsa-miR661-3p. The present study has great theoretical value for the complete interpretation of $\mathrm{UbcH} 10$ in the regulatory mechanism of NSCLC.

\section{Materials and methods}

Cell culture. Human NSCLC cells (A549, SK-MES-1 and NCI-H266) and immortalized human normal lung cells (BEAS-2B), were purchased from the Cell Bank of the Chinese Academy of Sciences (Shanghai, China). The lentivirus-producing cell line, 293TN, was purchased from System Biosciences (Palo Alto, CA, USA). All the cell lines were maintained in Dulbecco's minimum essential medium (DMEM; Invitrogen; Thermo Fisher Scientific, Inc., Waltham, MA, USA) supplemented with $10 \%$ fetal bovine serum (FBS; Invitrogen; Thermo Fisher Scientific, Inc.). Cells were passaged by $0.25 \%$ trypsin digestion (Invitrogen; Thermo Fisher Scientific, Inc.) and incubated in an atmosphere of 5\% $\mathrm{CO}_{2}$ at $37^{\circ} \mathrm{C}$.

Tumor tissues. Twelve pairs of NSCLC and adjacent normal tissues were obtained from the Shanghai Pulmonary Hospital between October 2016 and December 2017 (Table I). Written informed consents were received from the patients and the study was approved by the Ethics Committee of the Shanghai Pulmonary Hospital. Samples were rinsed with saline and transferred to 2-ml microtubes, labeled and maintained in liquid nitrogen subsequently. Total RNA and protein were extracted and used for determination of hsa-miR-661-3p and $\mathrm{UbcH} 10$ protein, respectively.

\section{Construction of the vectors}

Construction of the cDNA expression vector. The CDS sequence of human UbcH10 (NM_007019.2) was amplified by using the primers 5'-GGAATTCGCCACCATGGCTTC CCAAAACCGCG-3' and 5'-CGGGATCCTCAGGGCTCC TGGCTGGTG-3', which contain an EcoRI cutting site and Kozak sequence and a BamHI cutting site, respectively, with the cDNA prepared by reverse transcription of RNA isolated from 293T cells. The PCR product was digested and cloned into the pcDH1 lentiviral-expressing vector; the recombinant vector was named pcDH1-UbcH10.
Construction of the hsa-miR-661-3p expression vector. Human genomic DNA was extracted from 293 cells and used for amplification of the template of precursor sequence of hsa-miR-661-3p. The primers used were: 5'-GGAATTCTG GCATGCCATAGCAGCGCAG-3' and 5'-CGGGATCCC TCCCATCTAAGCTTCCCAAAGTGT-3'. The PCR product was digested using EcoRI and BamHI and ligated into the linear pcDH1 vector (System Biosciences) and transformed into DH5 $\alpha$ competent cells. The obtained vector was called the pcDH1-miR-661 vector. The products of the vectors were confirmed by DNA sequencing. Endotoxin-free DNA was prepared in all cases.

Construction of the luciferase reporter vector. The 3'-untranslated region (3'-UTR, 259 bp) of human UbcH10 was amplified from cDNA obtained through the reverse transcription of total RNA of 293 cells, with the following primers: 5'-GCTCTAGAGAAACCTACTCAAAGCAG-3' and 5'-GCT CTAGAACCACAGCTCAAGATAAA-3'. The amplification parameters were as follows: 32 cycles of denaturation at $95^{\circ} \mathrm{C}$ for $10 \mathrm{sec}$, annealing at $58^{\circ} \mathrm{C}$ for $30 \mathrm{sec}$ and extension at $72^{\circ} \mathrm{C}$ for $30 \mathrm{sec}$. The product was digested with $\mathrm{XbaI}$ and inserted into the pGL3-promotor vector (Promega, Madison, WI, USA). The seed region was mutated from 5'-CCCAGG C-3' to 5'-CGCCACG-3 by point mutation, and the resultant vectors were called pGL-wt-UbcH10 and pGL-mt-UbcH10, respectively.

Lentivirus packaging. One day before the transfection, 293TN cells were seeded into $10-\mathrm{cm}$ dishes. A total of $2 \mu \mathrm{g}$ of each shRNA or expression vector and $10 \mu \mathrm{g}$ of pPACK Packaging Plasmid Mix (System Biosciences) were co-transfected using Lipofectamine 2000 (Invitrogen; Thermo Fisher Scientific, Inc.) in accordance with the manufacturer's protocol. The medium was replaced with DMEM plus $1 \%$ FBS. After $48 \mathrm{~h}$, the supernatant was harvested and cleared by centrifugation at $5,000 \mathrm{x} \mathrm{g}$ at $4^{\circ} \mathrm{C}$ for $5 \mathrm{~min}$ and then passed through a $0.45-\mu \mathrm{m}$ polyvinylidene difluoride (PVDF) membrane (EMD Millipore, Billerica, MA, USA). The titer of the virus was determined by gradient dilution. The packaged lentiviruses were named Lv-miR-661 and Lv-UbcH10.

Assessment of hsa-miR-661 and UbcH10 protein level in NSCLC specimens and NSCLC cell lines. Twelve pairs of NSCLC and para-carcinoma tissues, as well as A549,

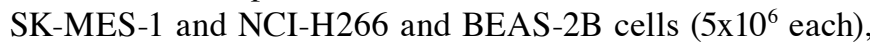
were collected, followed by total RNA extraction and real-time PCR for the measurement of hsa-miR-661-3p. Total protein extraction and western blotting was performed for the UbcH10 protein.

Effect of the expression of hsa-miR-661-3p on UbcH10. A549 and SK-MES-1 in logarithmic phase were seeded in 6 -well plates at $5 \times 10^{5}$ cells/well. One day later, a viral solution (Lv-miR-661 or Lv-UbcH10) was added at an MOI of 10. The infection efficiency was evaluated by observing and analyzing the fluorescence $72 \mathrm{~h}$ after infection. The total RNA was isolated from the cells and subjected to real-time PCR or western blotting for the level of hsa-miR-661-3p and UbcH10 protein, respectively. 
Table I. Clinicopathological features of 12 patients with NSCLC.

\begin{tabular}{rcclc}
\hline No. & Sex & $\begin{array}{c}\text { Age } \\
\text { (years) }\end{array}$ & \multicolumn{1}{c}{ Pathological } & $\begin{array}{c}\text { Clinical } \\
\text { stage }\end{array}$ \\
\hline 1 & M & 54 & Squamous carcinoma & II \\
2 & M & 60 & Squamous carcinoma & III \\
3 & F & 64 & Adenocarcinoma & IV \\
4 & M & 58 & Adenocarcinoma & IV \\
5 & F & 52 & Adenocarcinoma & III \\
6 & F & 68 & Squamous carcinoma & IV \\
7 & M & 70 & Squamous carcinoma & II \\
8 & M & 72 & Squamous carcinoma & IV \\
9 & F & 70 & Adenocarcinoma & III \\
10 & F & 69 & Adenocarcinoma & II \\
11 & F & 53 & Squamous carcinoma & III \\
12 & M & 73 & Adenocarcinoma & II \\
\hline
\end{tabular}

Twelve pairs of tumors of NSCLC and adjacent normal tissues were obtained from patients at Shanghai Pulmonary Hospital between October 2016 and December 2017. The clinical stage was determined according to the7th edition of TNM (UICC and AJCC). NSCLC, non-small cell lung cancer; $\mathrm{M}$, male; F, female.

Experiment of luciferase assay. We used Target Scan 7.1 (Whitehead Institute, Cambridge, MI, USA; http://www. targetscan.org/) to predict whether an hsa-miR661-3p binding site exists within the 3'-UTR of human UbcH10 mRNA. The results revealed that a seven-base hsa-miR-661-3p seed sequence is present in the 3'-UTR of UbcH10 mRNA. A suspension of 293T cells in logarithmic phase growth was prepared and the number of viable cells was counted using a hemocytometer in conjunction with trypan blue staining. The cells were seeded into 6-well plates at a concentration of $2 \times 10^{5}$ cells/well and maintained in Dulbecco's modified Eagle's medium supplemented with $10 \% \mathrm{FBS}$ at $37^{\circ} \mathrm{C}$ for $24 \mathrm{~h}$ in a $5 \% \mathrm{CO}_{2}$ atmosphere. The transfection of plasmid DNA and RNA was performed using Lipofectamine 2000 (Invitrogen; Thermo Fisher Scientific, Inc.). Transfection of cells with pGL-TK (100 ng) served as a reference for luciferase detection. Luciferase activity was assessed using the Dual-Luciferase reporter assay system (Promega) $48 \mathrm{~h}$ after transfection.

Cellular proliferation assay. We assessed whether regulation of has-miR-661-3p expression could inhibit the proliferation of NSCLC cell lines. A549 cells were infected with recombinant lentiviruses for $72 \mathrm{~h}$, trypsinized and seeded into 96-well plates at a density of $1 \times 10^{4}$ cells/well. The cells were cultured under normal conditions and cell viability was examined using CCK-8 (Cell Counting Kit-8; Dojindo Laboratories, Kumamoto, Japan) at 24, 48 and 72-h time-points. Briefly, $10 \mu 1$ of CCK-8 solution was added, and then the cells were cultured under normal conditions for an additional $4 \mathrm{~h}$ before measurement of the absorbance at $450 \mathrm{~nm}$. The experimental groups were as follows: i) Cell; ii) Cell+Lv-NC; iii) Cell+Lv-miR-661; and iv) Cell+Lv-miR-661+Lv-UbcH10.
Cell invasion assay. Cell invasion experiments were performed using the QCM ${ }^{\mathrm{TM}}$ 24-well Fluorimetric Cell Invasion Assay kit (ECM554; Chemicon International, Temecula, CA, USA) according to the manufacturer's instructions. The kit used an insert polycarbonate membrane with an $8-\mu \mathrm{m}$ pore size. The insert was coated with a thin layer of EC Matrix ${ }^{\mathrm{TM}}$ that occluded the membrane pores and blocked the migration of non-invasive cells. Culture medium $(500 \mu \mathrm{l})$ supplemented with $10 \%$ FBS was used as a chemoattractant. Cells that migrated and invaded the underside of the membrane were fixed in $4 \%$ paraformaldehyde. The invading cells were stained with crystal violet staining solution $(0.1 \%)$, and the number was then determined by fluorescence and reported as the relative fluorescence units (RFUs). SK-MES-1 cells were used and the grouping was the same as in the proliferation assay.

Effect of hsa-miR-661-3p on the expression of functional proteins of $S A C$. UbcH10, cyclin B, BubR1 and Mad2 protein levels in the A549 cells were assessed using western blotting $72 \mathrm{~h}$ after infection with Lv-NC, Lv-miR-661 or Lv-miR-661combined with Lv-UbcH0.

Cell cycle analysis. NCI-H266 cells were infected with Lv-miR-661-3p or Lv-UbcH10 as described above. The cells at logarithmic growth phase were typsinized, washed with phosphate-buffered saline (PBS) twice, and then fixed with $70 \%$ ethanol at $4^{\circ} \mathrm{C}$ overnight. The fixed cells were washed with PBS twice, resuspended in $100 \mu \mathrm{l}$ PBS [(containing $100 \mu \mathrm{g} / \mathrm{ml}$ ribonuclease $\mathrm{A}$ and $50 \mu \mathrm{g} / \mathrm{ml}$ propidium iodide (PI)], and incubated at room temperature for $30 \mathrm{~min}$. The cell suspensions were detected by a BD FACSCalibur flow cytometer (BD Biosciences, Franklin Lakes, NJ, USA).

Real-time PCR. Total RNA (2 $\mu \mathrm{g})$ was used for cDNA preparation using M-MLV reverse transcription kit and specific primers: U6 snRNA (NM_001101.3), 5'-TACCTTGCGAAG TGCTTAAAC-3' and miR-661, 5'-GTCGTATCCAGTGCG TGTCGTGGAGTCGGCAATTGCACTGGATACGATCGC G-3'. RNA contents were detected using PCR of fluorescent dye (Takara Bio Group, Beijing, China) in accordance with the manufacturer's instructions. The following primers were used for quantification of human U6 snRNA and miR-661: U6 snRNA, 5'-GTGCTCGCTTCGGCAGCACAT-3' and 5'-TAC CTTGCGAAGTGCTTAAAC-3', producing a segment of $112 \mathrm{bp}$; and miRNA631, 5'-GCCGGCGCCCGAGCTCTG GCTC-3' and 5'-TGCCTGGGTCTCTGGCCTGCGCGT-3', producing a segment of $72 \mathrm{bp}$. The PCR system included: Takara SYBR Premix Ex Taq $10 \mu$, forward and reverse primers $(20 \mu \mathrm{M}) 0.2 \mu \mathrm{l}$ each, and cDNA $2 \mu \mathrm{l}$, added with $\mathrm{dH}_{2} \mathrm{O}$ to $20 \mu \mathrm{l}$. Cycling parameters were as follows: 40 cycles of denaturation at $95^{\circ} \mathrm{C}$ for $10 \mathrm{sec}$, annealing at $60^{\circ} \mathrm{C}$ for $20 \mathrm{sec}$ and extension at $72^{\circ} \mathrm{C}$ for $20 \mathrm{sec}$. The levels of hsa-miR-661-3p were normalized using the $\Delta \Delta \mathrm{Ct}$ method; U6 snRNA was used as a reference. Each RNA sample was run in triplicate.

Detection of protein contents in the cells or tissues. The total protein was extracted from the cells using M-PER mammalian protein extraction reagent (Pierce, Rockford, IL, USA) or from tissues using T-PER tissue protein extraction reagent (Pierce). Equal amounts of protein ( $20 \mu \mathrm{g}$ per lane) estimated by the 
bicinchoninic acid (BCA) protein assay were loaded onto (11\%) SDS-PAGE gels and transferred onto nitrocellulose membranes. Blots were blocked in Tris-buffered saline (TBS) containing Tween-20 (TBST) containing 5\% non-fat milk at room temperature for $2 \mathrm{~h}$ and incubated with primary antibodies against human UbcH10 (dilution 1:200; cat. no. ab12290), cyclin B (dilution 1:500; cat. no. ab32053), BubR1 (dilution 1:600; cat. no. ab183496), Mad2 (dilution 1:250; cat. no. ab61591) and $\beta$-actin (dilution 1:1,200; cat. no. ab227387) (Abcam, Cambridge, UK), followed by the secondary HRP-conjugated anti-rabbit antibody (cat. no. ab97051; Abcam). Enhanced chemiluminescence (ECL) substrates (Pierce) and X-ray film were used to detect the bands, and the relative optical densities were analyzed using software Total Lab v1.10 (Total Lab Ltd., Newcastle, UK). $\beta$-actin was used as an endogenous reference for normalization.

Animal xenografts. Six- to eight-week old nude mice $(20 \pm 2 \mathrm{~g})$ were purchased from Shanghai Slack Experimental Animal Co., Ltd. (Shanghai, China) and housed at the Second Military Medical University Animal Experiment Center, where the implantation experiment was performed. All mice were bred and maintained in a specific pathogen-free facility and were used in accordance with the institutional guidelines for animal care and all the protocols were previously approved by the Tongji University Experiment Animal Ethics Committee. A549 cells $\left(1 \times 10^{5}\right)$ were suspended in $200 \mu 1$ medium, and injected subcutaneously into the flank regions of 48 female athymic nude mice. Two weeks after the inoculation, visible subcutaneous tumors were detected, and the tumors were $\sim 2.5 \mathrm{~mm}$ in diameter 3 weeks after inoculation. All animals were randomly divided into 4 groups (12 mice per group): The Model group, the Lv-control group, the miR-661 expression group and the miR-661-expression+UbcH10 overexpression group. For the intervention groups, each animal received $50 \mu 1$ recombinant lentivirus $\left(5 \times 10^{7}\right.$ IFU) twice a week (on Monday and Thursday) from the second week for 4 weeks, while the model group received the same volume of saline instead. The tumor diameter was assessed weekly from the second week, and the data were used to plot the tumor growth curves. The formula for calculating the tumor volume $(\mathrm{V})$ was: $\mathrm{V}=0.5 \mathrm{xaxbxb}$, where $\mathrm{a}$ and $\mathrm{b}$ are the long and short diameters of the tumor, respectively.

Statistical analysis. All data are expressed as the mean \pm SD and analyzed by Student's t-test or one-way analysis of variance (ANOVA) test. Least significant difference (LSD) was used for multiple comparisons between any two means. $\mathrm{P}<0.05$ was considered to indicate a statistically significant result. All statistical analysis was performed using SPSS 18.0 software (SPSS, Inc., Chicago, IL, USA).

\section{Results}

hsa-miR-661-3p is downregulated and UbcH1O is enhanced in NSCLC tumors and cell lines. The results of the hsa-miR-661-3p quantification in NSCLC showed that the expression of hsa-miR-661-3p was lower in NSCLC tumors than that in adjacent tissues $(\mathrm{P}<0.01)$, and the data of the UbcH10 protein evaluation indicated that the level of
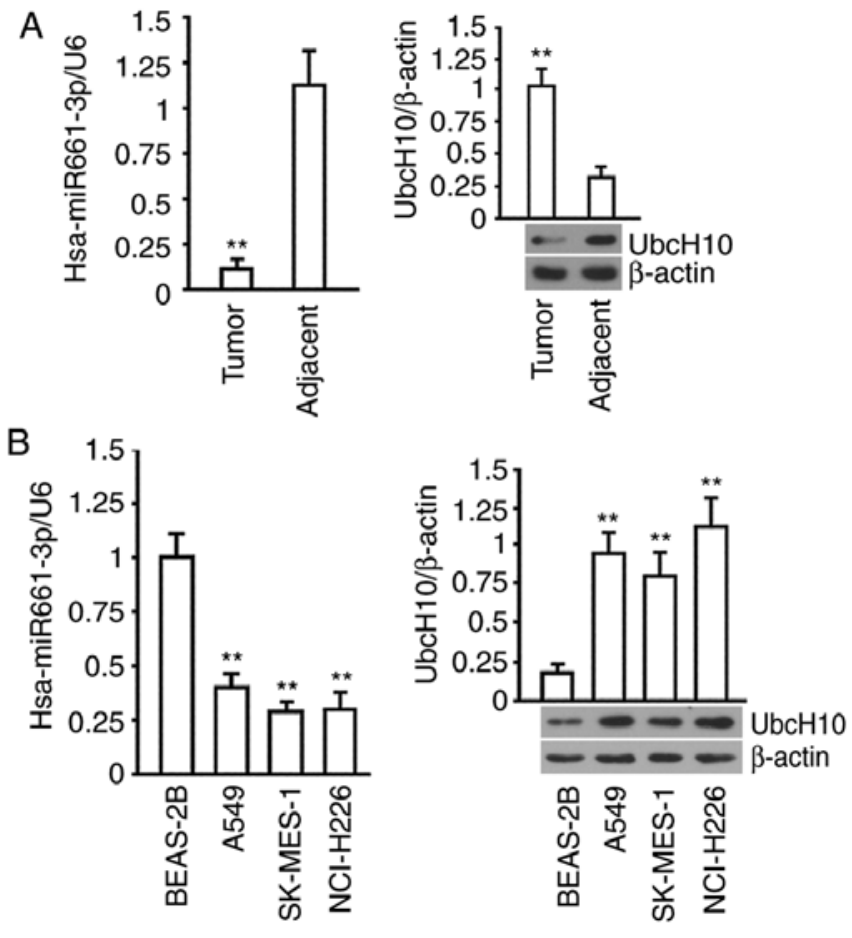

Figure 1. Levels of hsa-miR-661-3p and UbcH10 protein in NSCLC tissue and cells. (A) Determination of hsa-miR-661-3p and UbcH10 protein levels in 12 pairs of NSCLC tissues and adjacent tissues. U6 served as an internal reference for the determination of hsa-miR-661-3p, and the relative hsa-miR-6613p expression in NSCLC tissue was used for normalization in the comparison between groups (left image); for the determination of $\mathrm{UbcH} 10$ protein expression, 12 pairs of samples were pooled, and tested by western blotting, $\beta$-actin served as an internal reference, and the relative $\mathrm{UbcH} 10$ expression in the NSCLC samples were used for normalization in comparison between the groups (right image). ${ }^{* *} \mathrm{P}<0.01$ vs. adjacent tissue. (B) Determination of hsa-miR-661-3p (left) and $\mathrm{UbcH} 10$ protein (right image) in A549, SK-MES-1, NCI-H226 and BEAS-2B cells. U6 served as an internal reference for the determination of hsa-miR-661-3p, and the relative expression of hsa-miR-661-3p in cells was used for normalization in comparison among the groups; $\beta$-actin served as an internal reference for determination of the UbcH10 protein, and the relative expression of the $\mathrm{UbcH} 10$ protein in cells was used for normalization in comparison among the groups. ${ }^{* *} \mathrm{P}<0.01$ vs. BEAS-2B. The tests were carried out on three biological triplicates, and the data are expressed as the mean \pm SD.

UbcH10 protein was higher in NSCLC tumors than these levels in adjacent tissues $(\mathrm{P}<0.01)($ Fig. 1A). The examination in BEAS-2B, A549, SK-MES-1 and NCI-H226 cells revealed that hsa-miR-661-3p and UbcH10 protein were elevated in these cell lines compared with BEAS-2B, which is a normal lung cell line $(\mathrm{P}<0.01)$ (Fig. 1B). These results collectively suggested that the expression of hsa-miR-661-3p was negatively associated with $\mathrm{UbcH} 10$.

hsa-miR-661-3p inhibits UbcH10 expression by interacting with the 3'UTR of UbcH1O mRNA. Our bioinformatic analysis identified a seven-base hsa-miR-661-3p seed sequence in the 3'UTR of UbcH10 mRNA (Fig. 2A). Therefore, we constructed luciferase reporter vectors to verify whether this site represents a valid hsa-miR-661-3p target. Reporter vectors were generated that contained the wild-type UbcH10 3'-UTR or a variant in which the hsa-miR-661-3p target site within the 3'UTR had been mutated. Both reporter constructs expressed luciferase at a high level (Fig. 2A). However, the miR-661-3p 


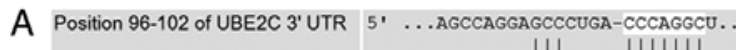
3 ha-miR-661
UGCGCGUCCGGUCUCUGGGUCCGU

B

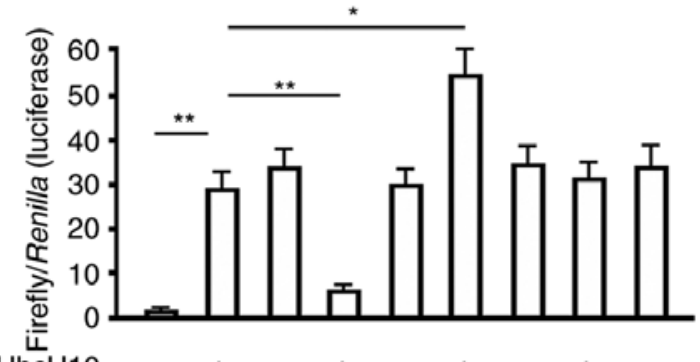

$\begin{array}{rrrrrrrrrr}\text { pGL3-wt-UbcH10 } & - & + & - & + & - & + & - & + & - \\ \text { pGL3-mt-UbcH10 } & - & - & + & - & + & - & + & - & + \\ \text { miR661 mimics } & - & - & - & + & + & - & - & - & - \\ \text { miR661 inhibitor } & - & - & - & - & - & + & + & - & - \\ \text { Negative control } & - & - & - & - & + & - & - & + & +\end{array}$

Figure 2. Hsa-miR-661-3p binds to UbcH10 3'UTR. (A) Predicted binding site of hsa-miR-661-3p in the 3'-UTR of the UbcH10 gene; (B) Effects of hsa-miR-661-3p on the expression of a luciferase cassette encoding the UbcH10 3'-UTR. Cells (293) were transfected with pGL3-wt-UbcH10 or pGL3-mt-UbcH10 in the presence or absence of miR-661-3p-mimics or inhibitor and subjected to a luciferase activity assay $48 \mathrm{~h}$ later. The histogram revealed the relative firefly luciferase activity for the different experimental groups. ${ }^{*} \mathrm{P}<0.05$ and ${ }^{* *} \mathrm{P}<0.01$ compared with the group transfected with the same vector but without the miR-661-3p mimics or miR-661-3p inhibitor. Data are expressed as the mean \pm SD of at least three independent experiments.

mimic significantly inhibited luciferase activity in cells transfected with the reporter vector encoding the wild-type 3'UTR $(27.21 \pm 3.61$ vs. $6.83 \pm 1.12 ; \mathrm{P}<0.01)$, while the miRNA-661-3p inhibitor significantly increased luciferase activity in these cells $(27.21 \pm 3.61$ vs. $52.67 \pm 9.04 ; \mathrm{P}<0.05)$. Conversely, in cells transfected with the reporter vector encoding the mutated hsa-miR-661-3p target site, neither the miR-661-3p mimic nor the miR-661-3p inhibitor had any observable effect on luciferase activity $(\mathrm{P}>0.05)$. Co-transfection of miR-661-3p-NC (non-targeting control) had no effect on the luciferase activity of either of the vectors $(\mathrm{P}>0.05)$. These results verified the presence of a hsa-miR-661-3p target site in the 3'UTR of UbcH10 mRNA and demonstrated that binding of hsa-miR-661-3p to this target site downregulated UbcH10 expression.

Effect of the expression of hsa-miR-661-3p and UbcH10 via lentiviral approach NSCLC cells. Recombinant lentiviruses, Lv-control, Lv-miR-661 and Lv-UbcH10, were used to infect A549 cells. GFP (Green fluorescent protein) was detected in most of the cells $72 \mathrm{~h}$ after infection, while the proportion of GFP-expressing cells suggested that the gene delivery efficiency was higher than $95 \%$ in the A549 (Fig. 3A). Hsa-miR-661-3p was significantly increased by Lv-miR-661 $(\mathrm{P}<0.01)$, and no change was observed in cells infected with Lv-UbcH10 $(\mathrm{P}>0.05)$. The protein level of UbcH10 was significantly increased by Lv-UbcH10 and decreased by Lv-miR-661 $(\mathrm{P}<0.01)$ (Fig. 3B). The same data were obtained in SK-MES-1 cells (data not shown). These findings indicated that decrease of hsa-miR-661-3p upregulated UbcH10 expression in A549 cells, and overexpression of UbcH10 had no obvious effect on hsa-miR-661-3p, thus, there is an obvious upstream and downstream relationship between hsa-miR-661-3p and $\mathrm{UbcH} 10$, that is to say, hsa-miR-661-3p is located upstream of the UbcH10.
A
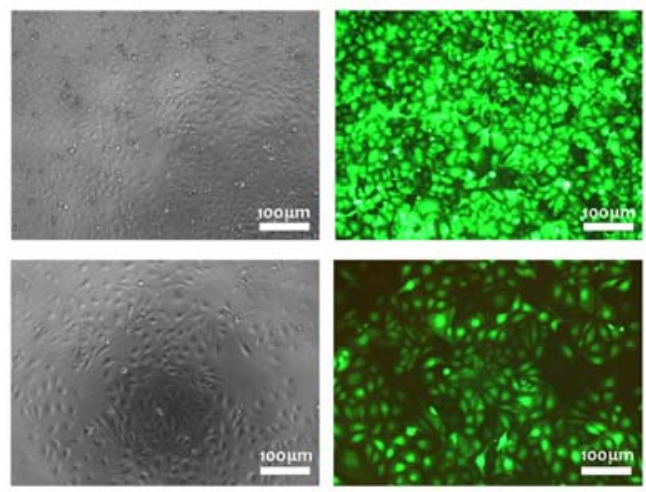

B
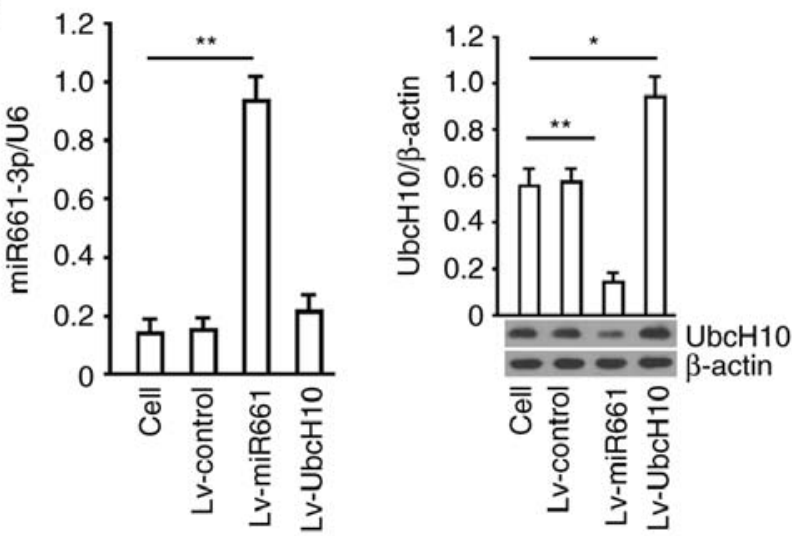

Figure 3. Genetic intervention through a lentiviral approach and interaction detection. (A) GFP expression $72 \mathrm{~h}$ after A549 was infected with a recombinant virus. The infection rate was estimated by dividing the number of the cells expressing GFP with the number of all the cells in each view. For statistical analysis, five views were randomly selected, and the mean was calculated. (B) Determination of hsa-miR-661-3p by Real-time PCR with U6 serving as an internal reference, and the $\mathrm{UbcH} 10$ protein level was detected by western blotting with $\beta$-actin serving as an internal reference. ${ }^{* *} \mathrm{P}<0.01$, vs the cell group. The tests were carried out on three biological triplicates, and the data are expressed as the mean $\pm \mathrm{SD}$. ${ }^{*} \mathrm{P}<0.05$ and ${ }^{* *} \mathrm{P}<0.01$ vs. cell group.

Overexpression of hsa-miR-661-3p inhibits cellular proliferation and invasion and induces cell cycle arrest in A549 cells. A549 cells infected with Lv-miR-661-3p or $\mathrm{Lv}-\mathrm{UbcH} 10$ were subjected to the proliferation assays. The results indicated that exogenous expression of hsa-miR-661-3p inhibited the proliferation of A549 cells after 48 or $72 \mathrm{~h}$ of incubation ( $\mathrm{P}<0.05$, vs. the cell group or NC group). Cells infected with Lv-UbcH10 combined Lv-miR-661-3p have no significant difference on the proliferation compared to cell group or $\mathrm{NC}$ group ( $\mathrm{P}>0.05$, vs. the cell group or $\mathrm{NC}$ group) (Fig. 4A). To investigate whether the inhibition of hsa-miR-661-3p on cell proliferation of SK-MES-1 was mediated by cell cycle alteration, cell cycle distribution was determined. The results revealed that in comparison with the cell group, hsa-miR-661-3p expression significantly increased the cell population at the $\mathrm{G} 2$ phase, from $0.92 \pm 0.07$ to $14.02 \pm 1.24 \%(\mathrm{P}<0.01)$ indicating that overexpression of hsa-miR-661-3p caused a cell cycle G2 arrest in A549 cells (Fig. 4B). Furthermore, cell invasion assay results demonstrated that hsa-miR-661-3p expression inhibited invasion in NCI-H226 cells $(\mathrm{P}<0.01)$, and UbcH10 overexpression could reverse the invasion inhibition caused by hsa-miR-661-3p expression $(\mathrm{P}<0.05)$ (Fig. 4C). 

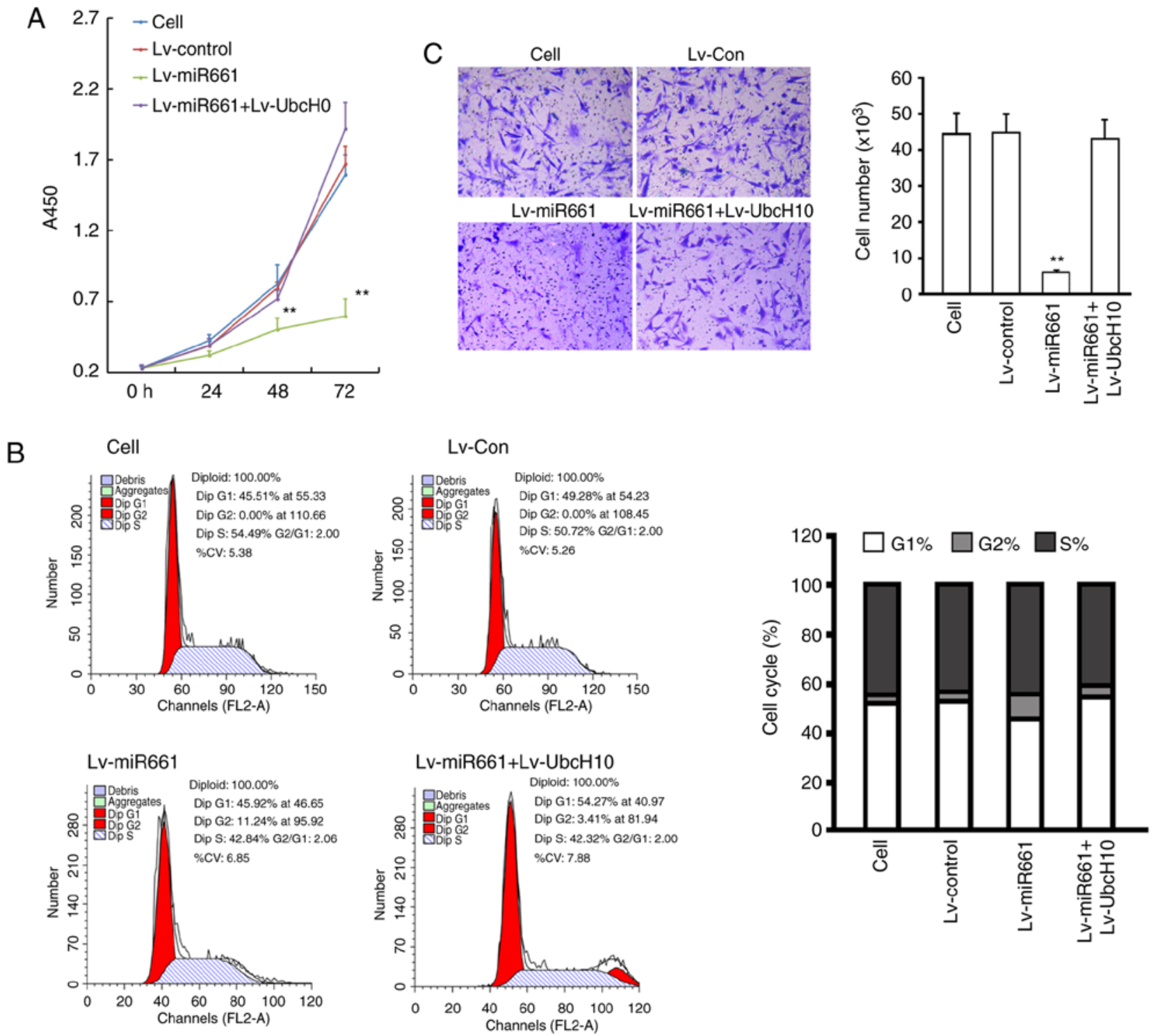

Figure 4. Effect of hsa-miR-661-3p depletion on the proliferation and invasion of A549 cells. (A) The proliferation of A549 cells 24-72 h after being infected with the indicated virus was determined by CCK-8 assay. The x-coordinate represents the cell grouping and the y-coordinate represents the absorbance at $450 \mathrm{~nm}$. (B) Cell cycle analysis produced by flow cytometry in NCI-H226 cells which were infected with a lentivirus. Cell cycle distribution in percentages of different groups. Data were obtained by flow cytometry. (C) Invasion data of the SK-MES-1 cells $72 \mathrm{~h}$ after being infected with the indicated virus determined by a Transwell assay. Crystal violet stained cells were the cells passing through the membrane, and the counts were the stained cells passing through the membrane, which were estimated using the absorbance and standard curve. The $\mathrm{x}$-coordinate represents the cell grouping and the $\mathrm{y}$-coordinate represents the cell number. ${ }^{* *} \mathrm{P}<0.01,{ }^{*} \mathrm{P}<0.05$ vs. the cell group. The tests were carried out on three biological triplicates, and the data are expressed as the mean $\pm \mathrm{SD}$. ${ }^{*} \mathrm{P}<0.05$ and ${ }^{* *} \mathrm{P}<0.01$ vs. cell group.

Effect of hsa-miR-661-3p on the expression of functional proteins of SAC. We assessed UbcH10, cyclin B, BubR1 and Mad2 in the hsa-miR-661-3p-overexpressed A549 cells. The results revealed that UbcH10 and cyclin B were decreased, while BubR1 and Mad2 were increased by hsa-miR-661-3p expression but not in the Lv-Control group (Fig. 5). These results indicated that hsa-miR-661-3p expression could regulate the expression of functional proteins of SAC by decreasing the expression of UbcH10 protein.

In vivo tumor suppression. An in vivo experiment revealed that 4 consecutive weeks of hsa-miR-661-3p expression significantly decreased the tumor volume. However, UbcH10 overexpression could significantly reverse the inhibition caused by hsa-miR-661-3p deficiency. After administration for four weeks, the tumor volume of the model group was $372.43 \pm 61.26 \mathrm{~mm}^{3}$, the Lv-control group was $424.94 \pm 56.59 \mathrm{~mm}^{3}$, the hsa-miR-661-3p expression group was $118.66 \pm 37.12 \mathrm{~mm}^{3}$, and the hsa-miR-661-3p and UbcH10 expression group was $633.31 \pm 83.02 \mathrm{~mm}^{3}$. The tumor inhibition rates in the control group, UbcH10 expression group and hsa-miR-661-3p and $\mathrm{UbcH} 10$ expression group were $0,68.12$ and $0 \%$, respectively, with a statistically significant difference between the miR-661-3p expression group and the other three groups $(\mathrm{P}<0.01)$ (Fig. 6).

\section{Discussion}

Since the 1980s, lung cancer has become the most malignant cancer with the highest morbidity and mortality rates 

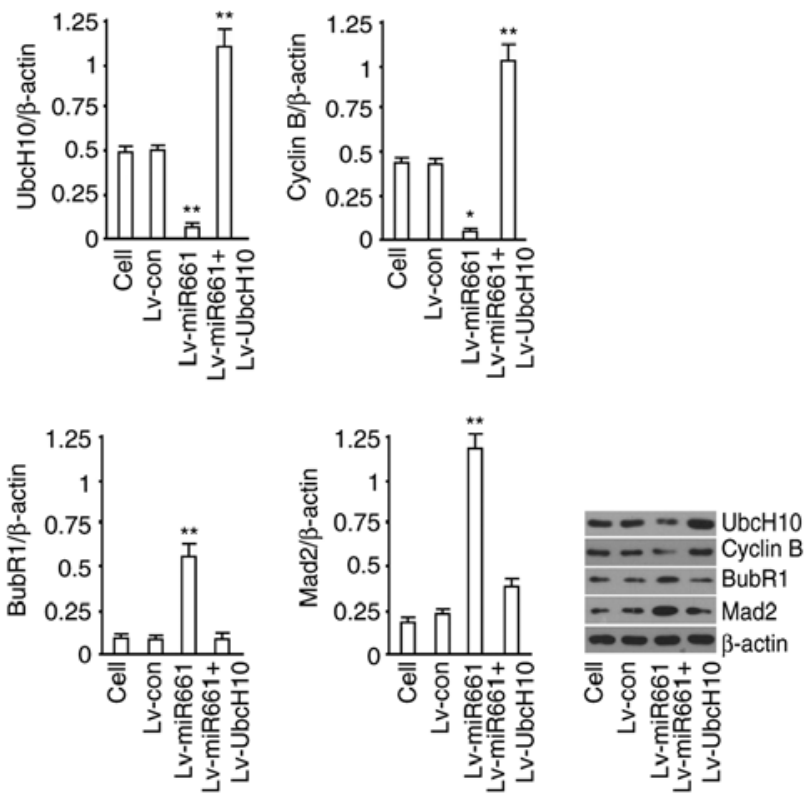

Figure 5. Effects of hsa-miR-661-3p expression on UbcH10, cyclin B, BubR1 and Mad-2. A549 cells infected with or without Lv-miR-661-3p or $\mathrm{Lv}$-UbcH10, $72 \mathrm{~h}$ post-infection were detected using western blotting. $\beta$-actin was used as a loading control. Data were representative of at least three independent experiments. ${ }^{*} \mathrm{P}<0.05$ and ${ }^{* *} \mathrm{P}<0.01$ vs. cell group.

worldwide. Statistics have revealed that the five-year survival rate of lung cancer patients in the United States is only $15 \%$, but less than $8.9 \%$ in developing countries (13). In China, more than 700,000 new cases of lung cancer are diagnosed each year, among them, non-small cell lung cancer (NSCLC) accounts for approximately $85 \%$ of the total cases (14). Therefore, a thorough understanding of the mechanism of pathogenesis of NSCLC is critical importance.

By comparing data of chromosomal status analysis in NSCLC patients, the proportion of chromosomal abnormalities could reach more than $40 \%$ compared with control subjects (14). The dysfunction of spindle assembly checkpoint (SAC) is primarily responsible for this phenomenon and it may be an important reason for the tumorigenesis of NSCLC (15-17). Some SAC member proteins such as BubR1, Mad2 are aberrantly expressed with the dysfunction of SAC and are related to lung cancer $(18,19)$. Unfortunately, the specific mechanism of chromosome aberration in NSCLC patients has not been clearly elucidated.

UbcH10 has been revealed to play an important role in the dysfunction of SAC in NSCLC cells. UbcH10 is a member of the ubiquitin binding enzyme gene family, and is a key member of the ubiquitin/proteasome protein degradation pathway (UPP) in the human body $(20,21)$. Okamoto et al firstly revealed that $\mathrm{UbcH} 10$ was upregulated in lung cancer tissues and cell lines (7). UbcH10 was a significant proto-oncogene that could cause genomic instability and cancer susceptibility, and $\mathrm{UbcH} 10$-overexpressing transgenic mice lung cancer was easily induced (14). In our previous study, we revealed that $\mathrm{UbcH} 10$ expression had a significant correlation with the pathological type and tissue differentiation of lung cancer. It was also significant negative correlation with the post-surgery survival time of patients with NSCLC $(\mathrm{P}=0.012)$. These suggests that similar to other cancers, UbcH10 may be used

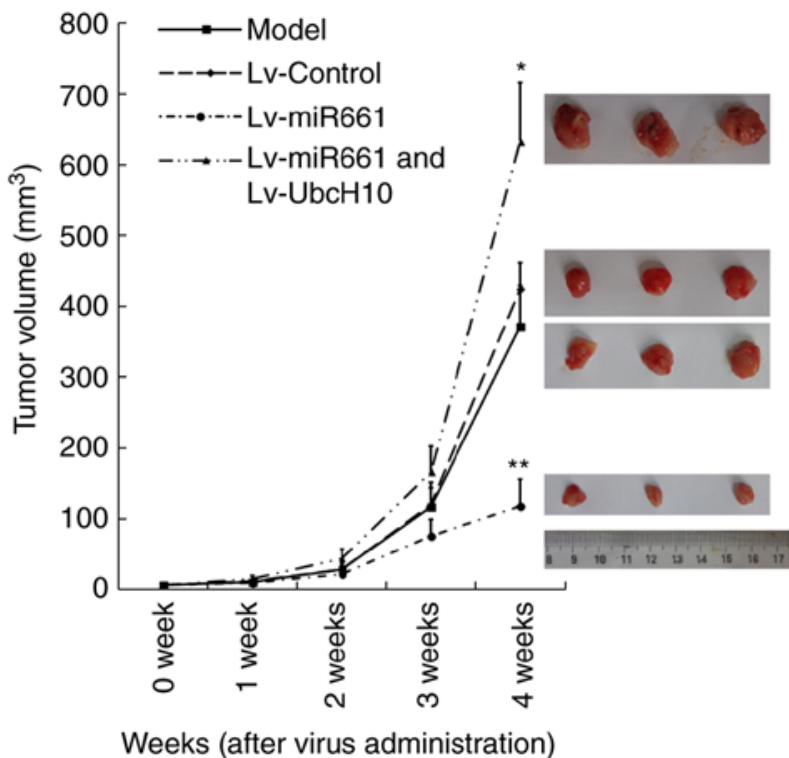

Figure 6. Growth curves of tumors in vivo. The $\mathrm{x}$-coordinate represents the period of injection and the $y$-coordinate represents the tumor volume. The formula for calculating the tumor volume was: $V=0.5 \times a x b x b$, where $a$ and $b$ are the long and short diameters of the tumor $(\mathrm{mm})$. The number of animals in each group was $12(\mathrm{n}=12) .{ }^{* *} \mathrm{P}<0.01,{ }^{*} \mathrm{P}<0.05$ vs. the modle group. Data are expressed as the means $\pm \mathrm{SD}$.

as a prognostic factor in patients with NSCLC (8). In addition, an in vitro study found that inhibition of UbcH10 expression increased the sensitivity of SK-MES-1 cells to chemotherapeutic drugs gemcitabine and paclitaxel, and the possible mechanism may be due to the inhibition of tumor cell proliferation and cell cycle change (8). Therefore, it was proposed that UbcH10 maybe play an essential role in the dysfunction of SAC in NSCLC cells through regulated mitosis.

In this study, we first detected and analyzed the high expression of UbcH10 in NSCLC in transcription or post transcription. The quantitative detection of the mRNA of the $\mathrm{UbcH} 10$ gene in NSCLC cells and clinical specimens revealed that the mRNA of the UbcH10 gene in NSCLC tissue and NSCLC cells was slightly higher than that of paracancerous tissue and normal lung epithelial cells (data not show). Concurrently, western blotting detection data revealed that the expression of UbcH10 protein in NSCLC tissue and NSCLC cells was significantly higher than that of the paracancerous and BEAS-2B cells. When comparing the difference of UbcH10 mRNA and protein expression with controls, it was clearly demonstrated that UbcH10 was highly expressed in the occurrence and development of NSCLC and it was inactivated by post-transcriptional regulation.

Post-transcriptional regulation is the regulation of gene expression after RNA transcription, which is one of the characteristics of eukaryotic gene expression (22). As one of the classical post-reduction regulation mechanisms, miRNA combines with the 3'UTR region of the target gene to inhibit protein translation, therefore, it has been a hot spot in cancer research in recent years. At present, there are approximately 1,000 types of miRNA in human cells (of which $>400$ have been confirmed), which only account for approximately $1 \%$ of the protein encoding genes, however, they regulate approximately $30 \%$ of gene expression. Approximately $50 \%$ 
of miRNAs are located in the fragile sites or regions of chromosomes which are amplified or missing in cancer $(23,24)$. Studies had confirmed that the expression profile of miRNAs is closely related to the type, progression, patient survival rate and prognosis of lung cancer. miRNAs could be used as cancer suppressors or cancer promoting factors to regulate cell proliferation, apoptosis, invasion, metastasis and angiogenesis (25).

Yanaihara et al used a miRNA microarray analysis method to compare the miRNA expression profiles of 104 NSCLC tissues with normal lung tissues, and the results revealed that 43 miRNAs had significant expression differences, 15 miRNAs were upregulated and 28 miRNAs were downregulated (26). These findings built the foundation for the research of miRNAs in lung cancer. Studies have revealed that 5-miRNAs (miR34c-5p, miR34a, miR25, miR191 and let-7a) could accurately classify lung adenocarcinoma and lung squamous cell carcinoma (27). By comparing the expression profiles of 122 NSCLC patients (62 squamous cell carcinomas and 60 adenocarcinomas), Lebanony et al found that the sensitivity of miR205 to squamous cell carcinoma was $96 \%$ and the specificity was $90 \%$ when distinguishing between lung squamous cell carcinoma and lung adenocarcinoma, which was a highly specific biomarker for lung squamous cell carcinoma (28). A study conducted by Lima Queiroz et al revealed that malate dehydrogenase $1(\mathrm{MdH} 1)$ was overexpressed in NSCLC tissue and it had important prognostic value. The authors screened miRNA for the 3'UTR region of the target MdH1 gene and the results revealed that miRNA126-5p expression could inhibit apoptosis by inhibiting the expression of $\mathrm{MdH}$, while the inhibition of mitochondrial respiratory disease ultimately induced apoptosis in NSCLC cell lines (29). Karagur et al demonstrated that the high expression of miR-200 could reduce the expression of $\mathrm{N}$-cadherin and vimentin by increasing the expression of E-cadherin and targeting ZEB1 to inhibit the ZEB1. EMT is considered to be an initiator of tumor malignancy and miR-200 was capable of impeding the EMT of NSCLC A549 cell line (30). Thus, we wondered whether the increase of aberrant expression for UbcH10 in NSCLC tissues and cells was caused by the inactivation of miRNA regulatory mechanisms. Fortunately, in this study we were convinced that the high expression of UbcH10 in NSCLC tissues and cells was due to decreased expression of hsa-miR-661-3p.

The UbcH10 gene 3'UTR region was predicted by the biological prediction software, and the content of miRNA in the NSCLC tissues and cells in the pre-ranked seed region was quantitatively analyzed. The results revealed that the content of hsa-miR661-3p in the NSCLC tissues and cells was negatively associated with the expression of the UbcH10 protein. The luciferase reporter gene experiment further confirmed that hsa-miR-661-3p could negatively regulate the expression of the target gene by the combination of the 3'UTR region of gene UbcH10. Hsa-miR-661-3p is located on human chromosome 8 . At present, there are only few studies of this miRNA and its relationship to cancer. A study by Ali et al revealed that the combined analysis of hsa-miR-661-3p and ATG-4B gene content could be a marker for clinical diagnosis and prognosis of liver cancer (31). In addition, it has been reported that hsa-miR-661-3p played an important role in the maintenance of redox and metabolic balance of intestinal cancer cells (32). In the present study, to the best of our knowledge, we are the first to report that hsa-miR-661-3p could affect the dysfunction of SAC by regulating the expression of $\mathrm{UbcH} 10$ as a target gene, while the dysfunction of SAC could lead to the separation of chromosomes, rendering cell proliferation uncontrollable. In vitro experiments revealed that the overexpression of hsa-miR-661-3p through a lentivirus system could effectively inhibit the proliferation of A549 cells, which invaded and blocked the cells in the G2 phase. The in vivo experimental data revealed that overexpression of hsa-miR-661-3p could effectively inhibit the proliferation activity of the subcutaneous tumor-bearing cancer of nude mice of A549 cells. The UbcH10 gene suppression experimental group revealed that the inhibitory effect of hsa-mi661-3p on NSCLC was achieved through the target gene UbcH10. The SAC member protein BubR1 (Mad3), Mad2 and cyclin B also relied on D-box and KEN-box competitively inhibiting the combination of $\mathrm{Cdc} 20$ and APC, which inhibit the activity of APC/C as a pseudo-substrate. If BubR1, Mad2 and cyclin B did not comply with the strict timing and $\mathrm{APC} / \mathrm{C}$ complex dissociation and that were degraded by the UbcH10-APC/C complex in advance, it could cause the dysfunction of SAC and the termination of the checkpoint, leading eventually to the abnormal separation of the sister chromosomes and the cells withdrawing from mitosis in advance (33-36).

The important significance of this study is that on the basis of the functional research performed earlier on $\mathrm{UbcH} 10$ in NSCLC, we conducted a preliminary analysis of the downstream route of action and the upstream factors of UbcH10. The decrease of the expression of hsa-mi-661-3p in the NSCLC tissues and cells directly led to the increase of the expression of the UbcH10 protein. UbcH10 could promote progression of NSCLC randomly occurring through cycle regulation, indicating that hsa-miR-661-3p may be a valuable target for the clinical treatment of NSCLC. Detection of BubR1, Mad2 and cyclin $\mathrm{B}$ also revealed that overexpression of $\mathrm{UbcH} 10$ in A549 cells could affect the function of the UbcH10-APC/C complex, leading to the early recognition and degradation of SAC member proteins BubR1 and Mad2 in the G2/M phase. This may cause the dysfunction of SAC and lead to chromosomal segregation. We believe that these data provided an important theoretical value for fully elucidating the molecular mechanism of UbcH10 regulation of NSCLC.

For biological experiments, the wider applicability of the study is important. Therefore, validating the results of this study in expanded NSCLC cell lines and tissues will be the first step for all following future study plans. Though this study has systematically demonstrated that overexpression of hsa-miR-661-3p can effectively inhibited the proliferation and metastasis of non-small cell lung cancer by inhibiting the expression of $\mathrm{UbcH} 10$, it did not elucidate the reasons for the decrease of hsa-miR-661-3p in non-small cell lung cancer, which required further study. In the future, the collection of a large number of NSCLC clinical tissue specimens is planned, in order to analyze and explain the reasons for the decrease in the expression of hsa-miR-661-3p in NSCLC from an epigenetic point of view. The upstream regulation of hsa-miR-6613p/UbcH10 will continue to be our research target that helps us confirm hsa-miR-661-3p as a possible biomarker for clinical screening and prognosis evaluation of NSCLC. 


\section{Acknowledgements}

The authors thank Dr Jun Wang for his technical support.

\section{Funding}

The present study was supported by the National Natural Sciences Fund Project of China (NSFC no. 81372529), the Natural Science Fund Project of Shanghai (13ZR1414400), the Innovation Fund Project of Education Commission in Shanghai (14ZZ079), and the Special issue of Military Medical of the Second Military Medical University (2012JS21).

\section{Availability of data and materials}

The datasets used and/or analyzed during the current study are available from the corresponding author on reasonable request.

\section{Authors' contributions}

JL, XG and FL made substantial contributions to the conception or design of the work, the acquisition, the analysis, or the interpretation of data for the work; they also drafted the manuscript or revised it critically for important intellectual content. ZR substantially contributed to the conception or design of the work, the acquisition, the analysis, or interpretation of data for the study; ML and LZ contributed substantially to the conception or design of the work, the acquisition, the analysis, or the interpretation of data for the work; they drafted the manuscript or revising it critically for important intellectual content and performed most of the research works and wrote the manuscript. All authors agreement to be accountable for all aspects of the work in ensuring that questions related to the accuracy or integrity of any part of the work are appropriately investigated and resolved. All authors read and approved the final manuscript.

\section{Ethics approval and consent to participate}

The study and use of human samples was approved by the Ethics Committee of Shanghai Pulmonary Hospital and written informed consents were obtained from all patients. All the protocols involving animal experiments were previously approved by the Tongji University Experiment Animal Ethics Committee.

\section{Patient consent for publication}

Not applicable.

\section{Competing interests}

The authors declare that they have no competing interests

\section{References}

1. Hoeijmakers JH: Genome maintenance mechanisms for preventing cancer. Nature 411: 366-374, 2001.

2. Blagosklonny MV and Pardee AB: Exploiting cancer cell cycling for selective protection of normal cells. Cancer Res 61: 4301-4305, 2001.
3. Berlingieri MT, Pallante P, Sboner A, Barbareschi M, Bianco M, Ferraro A, Mansueto G, Borbone E, Guerriero E, Troncone G and Fusco A: UbcH10 is overexpressed in malignant breast carcinomas. Eur J Cancer 43: 2729-2735, 2007.

4. Berlingieri MT, Pallante P, Guida M, Nappi C, Masciullo V, Scambia G, Ferraro A, Leone V, Sboner A, Barbareschi M, et al: $\mathrm{UbcH} 10$ expression may be a useful tool in the prognosis of ovarian carcinomas. Oncogene 26: 2136-2140, 2007.

5. Troncone G, Guerriero E, Pallante P, Berlingieri MT, Ferraro A, Del Vecchio L, Gorrese M, Mariotti E, Iaccarino A, et al: UbcH10 expression in human lymphomas. Histopathology 54: 731-740, 2009

6. Ieta K, Ojima E, Tanaka F, Nakamura Y, Haraguchi N, Mimori K, Inoue $\mathrm{H}$, Kuwano $\mathrm{H}$ and Mori M: Identification of over expressed genes in hepatocellular carcinoma, with special reference to ubiquitin-conjugating enzyme E2C gene expression. Int J Cancer 121: 33-38, 2007.

7. Okamoto Y, Ozaki T, Miyazaki K, Aoyama M, Miyazaki M and Nakagawara A: UbcH10 is the cancer-related E2 ubiquitin-conjugating enzyme. Cancer Res 63: 4167-4173, 2003.

8. Zhao L, Jiang L, Wang L, He J, Yu H, Sun G, Chen J, Xiu Q and $\mathrm{Li} \mathrm{B}$ : UbcH10 expression provides a useful tool for the prognosis and treatment of non-small cell lung cancer. J Cancer Res Clin Oncol 138: 1951-1961, 2012.

9. Carmichael JA, Wing-San Mak D and O'Brien MA: Review of recent advances in the treatment of elderly and poor performance NSCLC. Cancers (Basel) 10: E236, 2018.

10. Sharma N and Graziano S: Overview of the LUX-lung clinical trial program of afatinib for non-small cell lung cancer. Cancer Treat Rev 69: 143-151, 2018.

11. Bassanelli M, Sioletic S, Martini M, Giacinti S, Viterbo A, Staddon A, Liberati F and Ceribelli A: Heterogeneity of PD-L1 expression and relationship with biology of NSCLC. Anticancer Res 38: 3789-3796, 2018.

12. Rubin H: Cancer development: The rise of epigenetics. Eur J Cancer 28: 1-2, 1992.

13. Parkin DM, Bray F, Ferlay $\mathbf{J}$ and Pisani P: Global cancer statistics, 2002. CA Cancer J Clin 55: 74-108, 2005.

14. Tomasini R, Tsuchihara K, Tsuda C, Lau SK, Wilhelm M, Ruffini A, Tsao MS, Iovanna JL, Jurisicova A, Melino G and Mak TW: TAp73 regulates the spindle assembly checkpoint by modulating BubR1 activity. Proc Natl Acad Sci USA 106: 797-802, 2009.

15. van Ree JH, Jeganathan KB, Malureanu L and van Deursen JM: Overexpression of the $\mathrm{E} 2$ ubiquitin-conjugating enzyme UbcH10 causes chromosome missegregation and tumor formation. J Cell Biol 188: 83-100, 2010.

16. Draviam VM, Xie S and Sorger PK: Chromosome segregation and genomic stability. Curr Opin Genet Dev 14: 120-125, 2004.

17. Nasmyth K, Peters JM and Uhlmann F: Splitting the chromosome: Cutting the ties that bind sister chromatids. Science 288: $1379-1385,2000$.

18. Hu L, Liu X, Chervona Y, Yang F, Tang MS, Darzynkiewicz Z and Dai W: Chromium induces chromosomal instability, which is partly due to deregulation of BubR1 and Emi1, two APC/C inhibitors. Cell Cycle 10: 2373-2379, 2011.

19. Kato T, Daigo Y, Aragaki M, Ishikawa K, Sato M, Kondo S and Kaji M: Overexpression of MAD2 predicts clinical outcome in primary lung cancer patients. Lung Cancer 74: 124-131, 2011.

20. Doherty FJ, Dawson S and Mayer RJ: The ubiquitin-proteasome pathway of intracellular proteolysis. Essays Biochem 38: 51-63, 2002.

21. Summers MK, Pan B, Mukhyala K and Jackson PK: The unique $\mathrm{N}$ terminus of the UbcH10 E2 enzyme controls the threshold for APC activation and enhances checkpoint regulation of the APC. Mol Cell 31: 544-556, 2008.

22. Barad O, Meiri E, Avniel A, Aharonov R, Barzilai A, Bentwich I, Einav U, Gilad S, Hurban P, Karov Y, et al: MicroRNA expression detected by oligonucleotide microarrays: System establishment and expression profiling in human tissues. Genome Res 14: 2486-2494, 2004.

23. Lai EC, Tomancak P, Williams RW and Rubin GM: Computational identification of Drosophila microRNA genes. Genome Biol 4: R42, 2003.

24. Lee I, Ajay SS, Yook JI, Kim HS, Hong SH, Kim NH, Dhanasekaran SM, Chinnaiyan AM and Athey BD: New class of microRNA targets containing simultaneous 5'-UTR and 3'-UTR interaction sites. Genome Res 19: 1175-1183, 2009.

25. Gilad S, Lithwick-Yanai G, Barshack I, Benjamin S, Krivitsky I, Edmonston TB, Bibbo M, Thurm C, Horowitz L, Huang Y, et al: Classification of the four main types of lung cancer using a microRNA-based diagnostic assay. J Mol Diagn 14: 510-517, 2012. 
26. Yanaihara N, Caplen N, Bowman E, Seike M, Kumamoto K, Yi M, Stephens RM, Okamoto A, Yokota J, Tanaka T, et al: Unique microRNA molecular profiles in lung cancer diagnosis and prognosis. Cancer Cell 9: 189-198, 2006.

27. Landi MT, Zhao Y, Rotunno M, Koshiol J, Liu H, Bergen AW, Rubagotti M, Goldstein AM, Linnoila I, Marincola FM, et al: MicroRNA expression differentiates histology and predicts survival of lung cancer. Clin Cancer Res 16: 430-441, 2010.

28. Lebanony D, Benjamin H, Gilad S, Ezagouri M, Dov A Ashkenazi K, Gefen N, Izraeli S, Rechavi G, Pass H, et al: Diagnostic assay based on hsa-miR-205 expression distinguishes squamous from nonsquamous non-small-cell lung carcinoma. J Clin Oncol 27: 2030-2037, 2009.

29. Lima Queiroz A, Zhang B, Comstock DE, Hao Y, Eriksson M, Hydbring $\mathrm{P}$, Vakifahmetoglu-Norberg $\mathrm{H}$ and Norberg $\mathrm{E}$ : miR-126-5p targets malate dehydrogenase 1 in non-small cell lung carcinomas. Biochem Biophys Res Commun 499: 314-320, 2018.

30. Karagur ER, Ozay C, Mammadov R and Akca H: Anti-invasive effect of Cyclamen pseudibericum extract on A549 non-smal cell lung carcinoma cells via inhibition of ZEB1 mediated by miR-200c. J Nat Med 72: 686-693, 2018.
31. Ali MA, Matboli M, El-Khazragy N, Saber O, El-Nakeep S, Abdelzaher HM, Shafei AE and Mostafa R: Investigating miRNA-661 and ATG4-B mRNA expression as potential biomarkers for hepatocellular carcinoma. Biomark Med 12: 245-256, 2018.

32. Gómez de Cedrón M, Acín Pérez R, Sánchez-Martínez R, Molina S, Herranz J, Feliu J, Reglero G, Enríquez JA and Ramírez de Molina A: MicroRNA-661 modulates redox and metabolic homeostasis in colon cancer. Mol Oncol 11: 1768-1787, 2017.

33. Lara-Gonzalez P, Scott MI, Diez M, Sen O and Taylor SS: BubR1 blocks substrate recruitment to the $\mathrm{APC} / \mathrm{C}$ in a KEN-box-dependent manner. J Cell Sci 124: 4332-4345, 2011.

34. Rape M, Reddy SK and Kirschner MW: The processivity of multiubiquitination by the APC determines the order of substrate degradation. Cell 124: 89-103, 2006.

35. Townsley FM, Aristarkhov A, Beck S, Hershko A and Ruderman JV: Dominant-negative cyclin-selective ubiquitin carrier protein E2-C/UBCH10 blocks cells in metaphase. Proc Natl AcadSci USA 94: 2362-2367, 1997.

36. Patel D and McCance DJ: Compromised spindle assembly checkpoint due to altered expression of $\mathrm{UBCH} 10$ and $\mathrm{Cdc} 20$ in human papillomavirus type 16 E6- and E7-expressing keratinocytes. J Virol 84: 10956-10964, 2010. 